\title{
Search for new physics in beta-neutrino correlations with the WITCH spectrometer
}

\author{
D. Beck ${ }^{\mathrm{a}}$, F. Ames ${ }^{\mathrm{b}}$, M. Beck $^{\mathrm{a}}$, G. Bollen ${ }^{\mathrm{c}}$, B. Delauré ${ }^{\mathrm{a}}$, \\ J. Deutsch ${ }^{\mathrm{d}}$, J. Dilling ${ }^{\mathrm{e}}$, O. Forstner ${ }^{\mathrm{f}}$, T. Phalet ${ }^{\mathrm{a}}$, R. Prieels ${ }^{\mathrm{d}}$, \\ W. Quint ${ }^{\mathrm{e}}$, P. Schmidt ${ }^{\mathrm{b}}$, P. Schuurmans ${ }^{\mathrm{a}}$, N. Severijns ${ }^{\mathrm{a}}$, \\ B. Vereecke ${ }^{a}$, S. Versyck ${ }^{a}$ \\ and the EUROTRAPS Collaboration ${ }^{\mathrm{e}}$ \\ ${ }^{a}$ IKS, K.U.Leuven, Celestijnenlaan 200 D, B-3001 Leuven, Belgium \\ ${ }^{\mathrm{b}}$ Institut für Physik, Universität Mainz, D-55099 Mainz, Germany \\ ${ }^{\mathrm{c}}$ L.M.U. München, Sektion für Physik, D-85748 München, Germany \\ dIPN, UCL, Chemin du Cyclotron 2, B-1348 Louvain-la-Neuve, Belgium

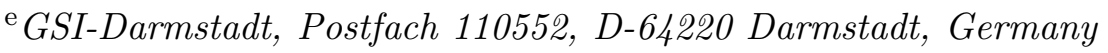 \\ ${ }^{\mathrm{f}} \mathrm{CERN}, \mathrm{CH}-1211$ Geneva, Switzerland
}

\begin{abstract}
The WITCH ${ }^{1}$ experiment is a retardation spectrometer coupled to a Penning trap and measures the beta-neutrino angular correlation via the shape of the recoil energy spectrum. The present form of the Standard Model describes weak processes in terms of vector and axial-vector type interactions, but the possible presence of scalar and tensor interactions is not yet ruled out. The main aim of this experiment is a test of the Standard Model for possible admixture of scalar and tensor currents.
\end{abstract}

Key words: Weak Interaction; Standard Model; Penning trap; Beta-Neutrino Angular Correlation

\section{Introduction}

The present form of the Standard Model of the electroweak interaction describes weak processes in terms of vector (V) and axial-vector (A) type interactions. Although there is strong experimental evidence for this $\mathrm{V}-\mathrm{A}$ form, the limits on possible admixture of scalar $(\mathrm{S})$ and tensor $(\mathrm{T})$ type interactions

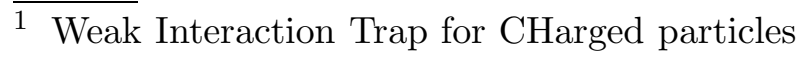


are still rather poor. Their coupling constants can be as large as $10 \%-15 \%$ (95\% CL) compared to the vector coupling constant $(1 ; 2 ; 3)$. Scalar or tensor couplings in the weak interaction would occur if yet unknown charged bosons or leptoquarks were exchanged instead of a $\mathrm{W}$ boson.

In nuclear beta decay scalar (tensor) interactions would show up most clearly in pure Fermi (Gamow-Teller) transitions through small anomalies. These can be revealed by determining the angular correlation (4) between the $\beta$-particle and the neutrino emitted by unpolarized nuclei,

$$
W(\Theta) \simeq 1+a \frac{v}{c} \cos (\Theta)\left[1-\frac{\Gamma m}{E} b\right]
$$

with $E, v / c$, and $m$ the total energy, the velocity and the rest mass of the $\beta$ particle, $\Gamma=\sqrt{1-(\alpha Z)^{2}}, b$ the Fierz interference coefficient, and $a$ the betaneutrino angular correlation coefficient. The coefficients $a$ and $b$ are linked to the coupling constants of the weak interaction as described in (4).

The observable measured in the WITCH experiment is the energy spectrum of recoil nuclei after beta decay (5). Figure 1 shows the correlation of the shape of the recoil spectrum to $a$ for ${ }^{46} \mathrm{~V}$ and ${ }^{35} \mathrm{Ar}$. Observing the recoil energy

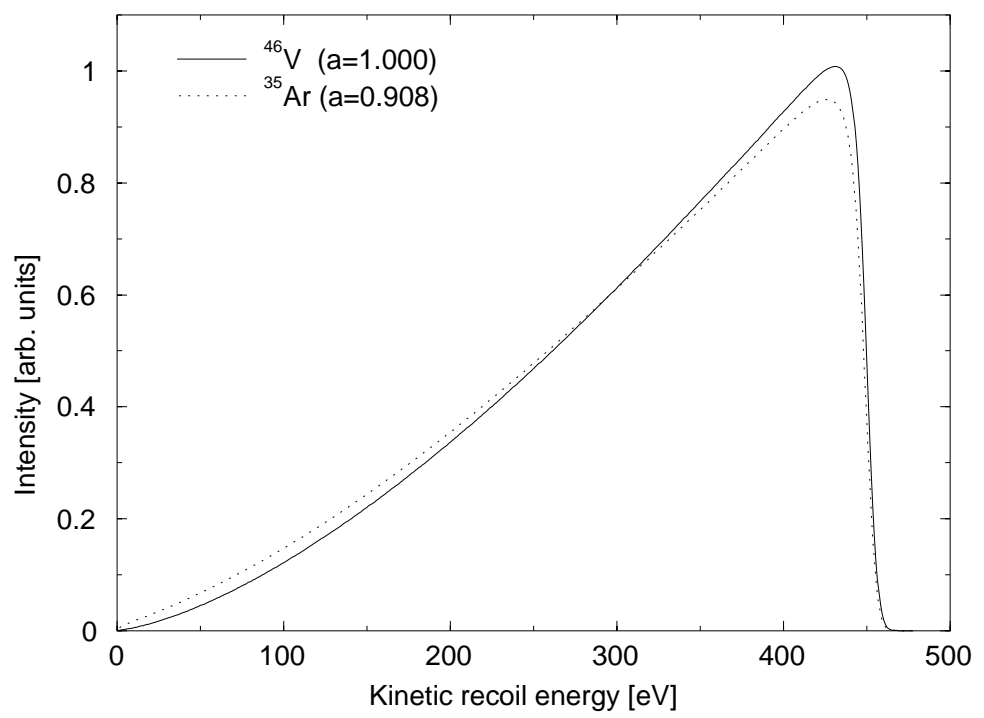

Fig. 1. Calculated recoil energy spectra following the $\beta$-decay of ${ }^{46} \mathrm{~V}$ (solid line) and ${ }^{35} \mathrm{Ar}$ (dashed line). The spectrum of ${ }^{46} \mathrm{~V}$ is scaled to the endpoint energy of ${ }^{35} \mathrm{Ar}$.

spectrum is made possible by using an ion trap. Here, the recoil particles do not scatter or lose energy compared to a radioactive source implanted into a matrix. Moreover, an electromagnetic trap is applicable to all elements and does not restrict the choice of the isotope investigated as atom traps do. The best limit on $a$ will be achieved with a measurement of a pure Fermi (GamowTeller) decay with only one decay channel to the ground state, e.g. ${ }^{46} \mathrm{~V}$. 


\section{Experiment}

Figure 2 shows a schematic view of the experiment. A Penning trap is lo-

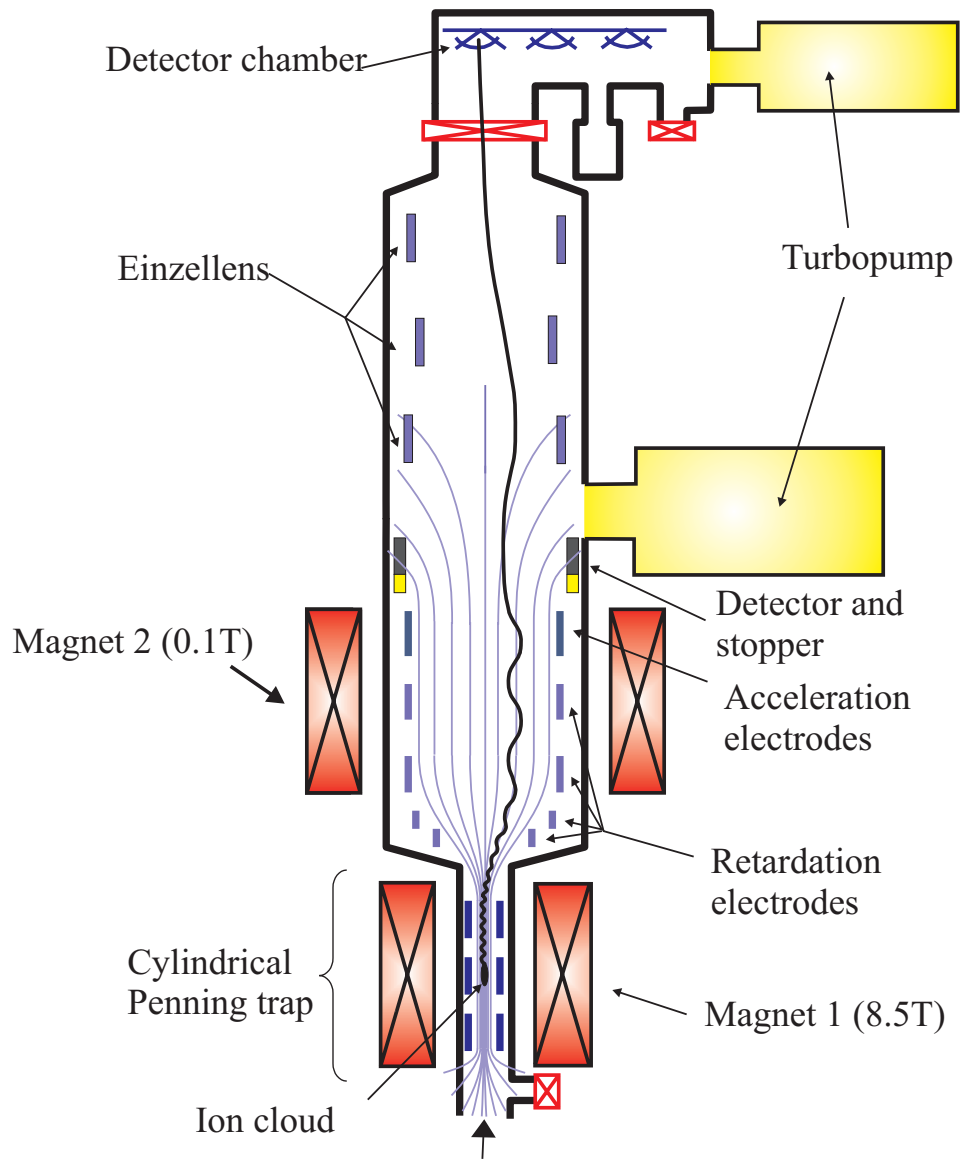

Fig. 2. Schematic layout of the WITCH spectrometer. The distance between the center of the Penning trap and the ion detector will be around $2.5 \mathrm{~m}$.

cated in the center of a superconducting magnet $(B \approx 8.5 \mathrm{~T})$ and coupled to a retardation spectrometer. The latter follows the same principle as the spectrometers used in Mainz and Troitsk $(6 ; 7)$. But instead of a second strong-field magnet an electrostatic beam transport system is used to focus the ions to the detector in the detector chamber. Electrodes around the analyzing plane, close to the center of the weak field magnet $(B \approx 0.1 \mathrm{~T})$, are used to create the electrostatic retardation potential. The energy resolving power of the spectrometer is $E / \Delta E \approx 100$.

A cloud of short-lived nuclei, that are produced by the on-line mass separator ISOLDE/CERN (8) and cooled and bunched by REXTRAP (9), is captured in-flight (10) and cooled via buffer gas collisions (11) in the trap. After decay, the recoil ions leave the trap spiraling around the magnetic field lines. While the ions drift from the strong to the weak magnetic field at the analyzing 
plane, their radial energy is converted into axial energy due to conservation of the magnetic moment of the ion's orbit. Then, their axial energy is analyzed with the electrostatic retardation potential. Finally, the ions are electrostatically accelerated to an energy of a few $\mathrm{keV}$ and focussed to the detector. By measuring the number of detected ions as a function of the retardation potential the integrated recoil energy spectrum can be measured.

\section{Status and time schedule}

Monte Carlo studies were performed to determine the statistical limit for the first experiment which will use the mixed transition ${ }^{35} \mathrm{Ar}$. Since the $\beta$-decay to the first excited state is sufficiently lower in energy, it will not disturb the measurement. The precision of the measured ft-value will lead to a systematic uncertainty of $0.3 \%$. The present experimental limits for the size of the scalar and tensor-type electroweak coupling constants can be reached after a few days of beam time as described in the accepted proposal (5). The design studies of the spectrometer are finished. Extensive calculations of ion trajectories were used to verify the properties of the spectrometer and to investigate systematic effects like the presence of buffer gas, the influence of the trap potential, and other effects on the shape of the energy spectrum. It should be noted that the set-up is versatile, so it can also be used for other experiments like the determination of Gamow-Teller to Fermi mixing ratios, $\beta$-decay to electron capture branching ratios or Q-value measurements. The purchasing of the equipment has started and first components are already being tested. The

spectrometer will be installed by beginning of the year 2001 and it is intended to perform first on-line tests by the end of the same year.

\section{References}

[1] C. H. Johnson et al., Phys. Rev. 132 (1963) 1149

[2] F. Glueck, Nucl. Phys. A 628 (1998) 493

[3] E. Adelberger et al., Phys. Rev. Lett. 83 (1999) 1299

[4] J. D. Jackson et al., Nucl. Phys. 4 (1957) 206

[5] D. Beck et al., proposal document, CERN/ISC99-13 ISC/P111

[6] A. Picard et al., Nucl. Instr. Meth. B 63 (1992) 345

[7] V. M. Lobashev and P. E. Spivak, Nucl. Instr. Meth. A 240 (1985) 305

[8] E. Kugler et al., Nucl. Instr. Meth. B 70 (1992) 41

[9] F. Ames et al., Proc. Exotic Nuclei and Atomic Masses (Bellaire, USA, 1998), A.I.P. Conf. Proc. 445 (1998) 927

[10] H. Schnatz et al., Nucl. Instr. Meth. A 251 (1986) 17

[11] G. Savard et al., Phys. Lett. A 158 (1991) 247 